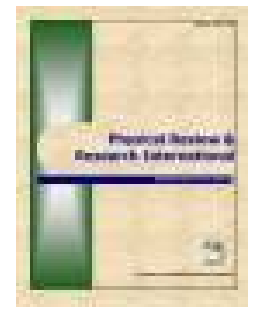

Physical Review \& Research International

3(4): 270-292, 2013

SCIENCEDOMAIN international

www.sciencedomain.org

\title{
Quantum Gravity and the Holographic Mass
}

\author{
Nassim Haramein ${ }^{1^{*}}$ \\ ${ }^{1}$ Director of Research, Hawaii Institute for Unified Physics, P.O. Box 1440, Kilauea, HI
} 96754.

Author's contribution

This work was carried out by author NH. Author $\mathrm{NH}$ read and approved the final manuscript.

Research Article

Received $21^{\text {st }}$ February 2013

Accepted 17 $7^{\text {th }}$ April 2013

Published 27 $7^{\text {th }}$ April 2013

\section{ABSTRACT}

We find an exact quantized expression of the Schwarzschild solution to Einstein's field equations utilizing spherical Planck units in a generalized holographic approach. We consider vacuum fluctuations within volumes as well as on horizon surfaces, generating a discrete spacetime quantization and a novel quantized approach to gravitation. When applied at the quantum scale, utilizing the charge radius of the proton, we find values for the rest mass of the proton within $0.069 \times 10^{-24} \mathrm{gm}$ of the CODATA value and when the 2010 muonic proton charge radius measurement is utilized we find a deviation of $0.001 \times 10^{-24} \mathrm{gm}$ from the proton rest mass. We identify a fundamental mass ratio between the vacuum oscillations on the surface horizon and the oscillations within the volume of a proton and find a solution for the gravitational coupling constant to the strong interaction. We derive the energy, angular frequency, and period for such a system and determine its gravitational potential considering mass dilation. We find the force range to be closely correlated with the Yukawa potential typically utilized to illustrate the exponential drop-off of the confining force. Zero free parameters or hidden variables are utilized.

Keywords: Quantum gravity; holographic principle; Schwarzschild solution; proton charge radius; strong interaction; Yukawa potential. 


\section{INTRODUCTION}

In 1916, Karl Schwarzschild published an exact solution to Einstein's field equations for the gravitational field outside a spherically symmetric body [1,2]. The Schwarzschild solution determined a critical radius, $r_{s}$ for any given mass where the escape velocity equals $c$, the speed of light. The region where $r=r_{s}$ is typically denoted as the horizon or event horizon and is given by the well known definition

$$
r_{s}=\frac{2 G m}{c^{2}}
$$

where $G$ is the gravitational constant, and $m$ is the mass. John Archibald Wheeler in 1967 described this region of space as a "black hole" during a talk at the NASA Goddard Institute of Space Studies. In 1957 Wheeler had already, as an implication of general relativity, theorized the presence of tunnels in spacetime or "wormholes" and in 1955, as a consequence of quantum mechanics, the concept of "spacetime foam" or "quantum foam" as a qualitative description of subatomic spacetime turbulence [3]. The theory predicts that the very fabric of spacetime is a seething foam of wormholes and tiny virtual black holes at the Planck scale as well as being the source of virtual particle production. In Wheeler's own words: "The vision of quantum gravity is a vision of turbulence - turbulent space, turbulent time, turbulent spacetime... spacetime in small enough regions should not be merely "bumpy," not merely erratic in its curvature; it should fractionate into ever-changing, multiplyconnected geometries. For the very small and the very quick, wormholes should be as much a part of the landscape as those dancing virtual particles that give to the electron its slightly altered energy and magnetism [Observed as the Lamb shift]." [4]

On the cosmological scale, black hole singularities were initially thought to have no physical meaning and probably did not occur in nature. As general relativity developed in the late $20^{\text {th }}$ century it was found that such singularities were a generic feature of the theory and evidence for astrophysical black holes grew such that they are now accepted as having physical existence and are an intrinsic component of modern cosmology. While the Schwarzschild solution to Einstein's field equations results in extreme curvature at the origin and the horizon of a black hole, it is widely utilized to give appropriate results for many typical applications from cosmology to planetary physics. For instance, the Newtonian gravitational acceleration near a large, slowly rotating, nearly spherical body can be derived by $g=r_{s} c^{2} / 2 r^{2}$ where $g$ is the gravitational acceleration at radial coordinate $r, r_{s}$ is the Schwarzschild radius of a gravitational central body, and $c$ is the speed of light. Similarly, Keplerian orbital velocity can be derived for the circular case by

$$
v=\sqrt{\frac{r_{s} c^{2}}{2 r}}
$$

where $r$ is the orbital radius. This can be generalized to elliptical orbits and of course the Schwarzschild radius is utilized to describe relativistic circular orbits or photon spheres for rapidly rotating objects such as black holes. There are many more examples of the ubiquitous nature of the Schwarzschild solution and its applications to celestial mechanics and cosmology. 
In developments over the past decade event horizons have been demonstrated to be dynamically fluctuating regions at a scale where quantum mechanical effects occupy a central role. Early explorations of spacetime fluctuations at the quantum level predicted that the vacuum at those scales undergoes extreme oscillations as formulated in the Wheeler model. Indeed, in quantum field theory, the vacuum energy density is calculated by considering that all the vibrational modes have energies of $\hbar \omega / 2$. When summed over all field modes, an infinite value results unless renormalized utilizing a Planck unit cutoff [5]. Yet, while the high curvature of general relativity and the vacuum fluctuations of quantum field theory converge and meet at the Planck cutoff, efforts to define gravitational curvature in a discrete and elegant manner, as in quantum gravity have proven elusive.

In the early 1970s, expanding from Hawking temperature theorems for black hole horizons, Bekenstein conjectured that the entropy of a black hole is proportional to the area of its event horizon divided by the Planck area times a constant on the order of unity [6]. Hawking confirmed Bekenstein's conjecture utilizing the thermodynamic relationships between energy and temperature [7].

$$
S=\frac{k A}{4 \ell^{2}}
$$

where $A$ is the area of the event horizon, $k$ is Boltzmann's constant, and $\ell$ is the Planck length. The Bekenstein bound conjecture and the entropy of a black hole eventually led to the holographic principle (generally given as an analogy to a hologram by Gerard 't Hooft) [8] where the covariant entropy bound demands that the physics in a certain region of space is described by the information on the boundary surface area, where one bit is encoded by one Planck area $[8,9]$. Since the temperature $T_{H}=\frac{k}{2 \pi}$ determines the multiplicative constant of the Bekenstein-Hawking entropy of a black hole which is

$$
S=\frac{A}{4}
$$

therefore, Hawking fixes the proportionality constant at $1 / 4$ of the surface area, which we note is equivalent to the surface area of the equatorial disc of the system.

In this paper, we generalize the holographic principle by utilizing a spherical Planck unit rather than a surface area Planck unit, $\ell^{2}$ as a minimum-size vacuum energy oscillator on which information encodes, which we term "Planck spherical unit" (PSU). This approach is consistent with the dimensional reduction of the holographic principle, which states explicitly that all the information of the interior volume of a black hole is encoded holographically on it's horizon surface. We consider the interior vacuum energy density ratio, in terms of PSU packing, to the surface horizon and find a generalized holographic principle which broadens the applicability of the holographic method to other areas of physics, such as gravitation, hadronic mass, and confinement.

As a result, an exact quantized derivation of the Schwarzschild solution to Einstein's field equations is found, yielding a novel approach to quantum gravity. We apply this method to 
the quantum scale and derive the proton rest mass from geometric considerations alone. When the CODATA charge radius value of the proton is employed, our result yields a very close first-order approximation within $\sim 4 \%$ deviation from the CODATA mass value, the difference of which is $0.069 \times 10^{-24} \mathrm{gm}$. Utilizing the 2010 muonic measurement of the proton charge radius however [10], we obtain a more accurate value within $0.001 \times 10^{-24} \mathrm{gm}$ or $\sim 0.07 \%$ deviation. Employing our generalized holographic approach we predict a precise proton charge radius. Our prediction falls within the reported experimental uncertainty for the muonic measurement of the proton charge radius [10].

By further algebraic derivation, we find a fundamental constant we term $\phi$, defined by the mass ratio of vacuum oscillations on the surface horizon to the ones within the volume of the proton. As a result, clear relationships emerge between the Planck mass, the rest mass of the proton, and the Schwarzschild mass of the proton or what we term the holographic gravitational mass. Further, we find that our derived fundamental constant $4 \phi^{2}$ generates the gravitational coupling constant to the strong interaction, thus defining the unification energy for confinement. We also derive the energy, angular frequency, and period for such a system utilizing our generalized holographic approach. We find that the period is on the order of the interaction time of particle decay via the strong force which is congruent with our derivation of the gravitational coupling constant. Moreover, the frequency of the system correlates well with the characteristic gamma frequency of the nucleon decay rate. Finally, we compute the gravitational potential resulting from the mass dilation of the system due to angular velocities as a function of radius and find that the gravitational force of such a system produces a force range drop-off closely correlated with the Yukawa potential typically utilized to define the short range of the strong interaction.

We demonstrate that a quantum gravitational framework of a discrete spacetime defined by spherical Planck vacuum oscillators can be constructed which applies to both cosmological and quantum scales. Our generalized holographic method utilizes zero free parameters and is generated from simple geometric relationships and algebra, yielding precise results for significant physical properties such as the mass of black holes, the rest mass of the proton, and the confining nuclear force.

Note that in this paper, we utilize the full significant digits of the Planck length and other relevant physical constants as given by CODATA in our derivations to demonstrate the accuracy of our results.

\section{THE SCHWARZSCHILD SOLUTION FROM PLANCK OSCILLATOR SPHERICAL UNITS}

In view of the increasingly significant role that quantum field effects or vacuum fluctuations have played in current cosmology to characterize the information structure of the horizons of astrophysical black holes, as in the holographic principle and its application to entropy [11], we examine a hypothetical black hole horizon of the approximate order of magnitude of the well documented black hole Cygnus $X-1$ with a radius of $\sim 2.5 \times 10^{6} \mathrm{~cm}$.

In order to better represent the natural systems of harmonic oscillators we initiate our calculation by defining a Planck spherical unit (PSU) oscillator of the Planck mass $m_{\ell}$ with a 
spherical volume $V_{\ell s}$ and a Planck length diameter $\ell=1.616199 \times 10^{-33} \mathrm{~cm}$ with a radius of $\ell_{r}=\ell / 2$. We utilize a spherical volume for our fundamental spacetime quantum foam PSU oscillator instead of the typical Planck area $\ell^{2}$ or Planck volume $\ell^{3}$ in our generalized holographic approach. Therefore a spherical PSU of radius $\ell_{r}$ has a volume of

$$
V_{\ell s}=\frac{4}{3} \pi \ell_{r}^{3}
$$

or $V_{\ell s}=2.210462 \times 10^{-99} \mathrm{~cm}^{3}$. Such a sphere will have an equatorial plane circular area of

$$
A_{\ell c}=\pi \ell_{r}^{2}
$$

or $A_{\ell c}=2.051538 \times 10^{-66} \mathrm{~cm}^{2}$, which will be utilized for the purpose of holographic tiling. In our generalized holographic approach we consider the volume vacuum oscillation energy in terms of Planck spherical units as well as the typical tiling of the surface horizon found in the holographic principle entropy calculations of equations (3) and (4). Our considerations of information within the volume stems from an exploration of the role of vacuum fluctuations in surface gravity and spacetime quantization relationships between the interior information network and the external surface tiling. It is important to note that although, in this exercise, we tile the surface horizon with Planck circular areas, these are equatorial areas of spherical oscillators.

Consequently, we derive the quantity $\eta$, the number of Planck areas $A_{\ell c}$ on the surface $A$ of the horizon of Cygnus $X-1$ with a radius of $2.5 \times 10^{6} \mathrm{~cm}$ and find that

$$
\eta=\frac{A}{A_{\ell c}}
$$

or $\eta=3.828339 \times 10^{79}$. We calculate $R$ or the quantity of Planck volume oscillators $V_{\ell s}$ within the volume $V$ of the interior of the Cygnus X-1 black hole

$$
R=\frac{V}{V_{\ell s}}
$$

or $R=2.960912 \times 10^{118}$. We then examine the relationship between the information network of the horizon $\eta$ and the interior information network of PSU oscillators $R$, then multiply it by the Planck mass, $m_{\ell}$ to obtain the mass-energy equivalence of the ratio and we determine that

$$
m_{h}=\frac{R}{\eta} m_{\ell}
$$


where $m_{h}=1.683354 \times 10^{34} \mathrm{gm}$ is the mass derived from this geometric approach, or what we term the "holographic gravitational mass". This expression can be written as well in terms of mass relations by multiplying equation (9) by $m_{\ell} / m_{\ell}$

$$
m_{h}=\frac{R_{\rho}}{\eta_{\rho}} m_{\ell}
$$

where $R_{\rho}$ is the total mass-energy of PSU oscillators within the volume and $\eta_{\rho}$ is the massenergy of PSU oscillators on the surface horizon, so that all terms are Planck mass quantities, which clarifies the relationship between masses in the geometry. Equation (10) can then be written as

$$
m_{h}=\frac{R_{\rho}}{\eta} .
$$

We then calculate the Schwarzschild mass of a black hole of the same radius as our example Cygnus X-1. Rearranging equation (1) we have

$$
\frac{r c^{2}}{2 G}=m_{s}
$$

where $m_{s}$ is the Schwarzschild mass of such a black hole, $c$ is the speed of light and $G$ is the gravitational constant. We obtain the exact same quantity, $m_{s}=1.683354 \times 10^{34} \mathrm{gm}$ utilizing CODATA values. Therefore

$$
m_{h}=m_{s} .
$$

We find that a simple relationship of the internal PSUs within a given volume, to the discrete "pixelation" of the holographic membrane surface horizon of the black hole yields what we term the holographic gravitational mass of the object which is equivalent to its classical Schwarzschild mass. This of course, is valid for any system, is free of any relativistic expressions, and utilizes only discrete Planck quantities, which has implications to quantum gravity.

From the above geometric analysis we then perform an algebraic derivation to find an elegant formulation of this quantized relationship. Therefore we can write equation (11) in terms of equation (7) and $R$

$$
\frac{R_{\rho}}{\eta}=\frac{R m_{\ell}}{A / A_{\ell c}}=\frac{R m_{\ell} A_{\ell c}}{A} .
$$

Utilizing equations (6) and (8) and rearranging terms we have 


$$
=\frac{\left(V / V_{\ell s}\right) m_{\ell} \pi \ell_{r}^{2}}{4 \pi r^{2}}=\frac{\left(V / V_{\ell s}\right) m_{\ell} \ell_{r}^{2}}{4 r^{2}} .
$$

Expanding to the spherical form in terms of $r$ and $\ell_{r}$ and reducing,

$$
=\frac{\left(4 / 3 \pi r^{3} /\left(4 / 3 \pi \ell_{r}^{3}\right)\right) m_{\ell} \ell_{r}^{2}}{4 r^{2}}=\frac{\left(r^{3} / \ell_{r}^{3}\right) m_{\ell} \ell_{r}^{2}}{4 r^{2}}
$$

or,

$$
\frac{R_{\rho}}{\eta}=r \frac{m_{\ell}}{4 \ell_{r}}
$$

where $r$ is the radius of a system. Given that $\ell_{r}=\ell / 2$, and utilizing equation (11) we now obtain what we have previously termed the holographic gravitational mass $m_{h}$ as,

$$
r \frac{m_{\ell}}{2 \ell}=m_{h}
$$

Of course now a radius we term the holographic radius $r_{h}$ can be calculated for any mass $m$, giving the expression

$$
r_{h}=2 \ell \frac{m}{m_{\ell}}
$$

Therefore, we find that the number of discrete Planck masses within any given mass $m$ multiplied by $2 \ell$, which is a discrete quantity, will generate the holographic radius equivalent to the well known Schwarzschild radius of equation (1) so that in the case of equation (19) we have a non-relativistic form derived from discrete vacuum oscillator Planck quantities generating a quantized solution. The geometric equation (9) and the algebraic derivation (19) are both simple and meaningful as they clearly demonstrate that the gravitational mass of an object can be obtained from discrete quantities based on Planck spherical units. Consequently our results are consistent with the dimensional reduction embodied in the holographic principle, and thus we have found a unique expression involving the holographic gravitational mass, radius, Planck mass, and the mass of any black-hole object that is congruent with the usual holographic entropy computation of equations (3) and (4).

Clearly in both cases $c$ and $G$ are involved since Planck entities are derived from $\ell=\sqrt{\frac{\hbar G}{c^{3}}}$ and $m_{\ell}=\sqrt{\frac{\hbar c}{G}}$, therefore we can write equation (19) as 


$$
r_{h}=2 m \frac{\ell}{m_{\ell}}=2 m \frac{\sqrt{\frac{\hbar G}{c^{3}}}}{\sqrt{\frac{\hbar c}{G}}}=2 m \sqrt{\frac{G^{2}}{c^{4}}}
$$

or

$$
r_{s}=r_{h}=\frac{2 G m}{c^{2}}
$$

Here we arrive to the Schwarzschild expression of equation (1) from geometric considerations alone. It then follows that the Schwarzschild solution to Einstein's field equations could have been developed in the late $19^{\text {th }}$ Century by computation of tiling Planck quantities independent of spacetime curvature and singularities, near the time when Max Planck in 1899 derived his units. His units were, of course, the result of the renormalization of the electromagnetic spectrum of black body radiation by the utilization of a quantum of action $h$, which confirmed experimental results. Planck quantities are natural units, free of any arbitrary anthropocentric measurements, are based on fundamental physical constants, and can be defined as, for example, the time it takes a photon to travel one Planck length which is the Planck time. Therefore, in the case of the generalized holographic solution the difficulties associated with discontinuities and singularity production are precluded from occurring due to the Planck quantization where the presence of $\hbar$, the quantum of angular momentum or the quantum of action of the energetic vacuum quantizes spacetime and yields a discrete gravitational mass or quantum gravity.

However, if our holographic solution is a correct representation of quantum gravitational spacetime structure, then it should be applicable to the quantum world and yield appropriate results such as fundamental physical quantities from first principles and geometric considerations.

\section{HOLOGRAPHIC MASS AT THE HADRON SCALE}

We now apply the above surface to volume relationships of Planck vacuum oscillations of a cosmological scale object to the quantum world. We initially utilize the standard CODATA proton charge radius given as $r_{p}=0.8775 \times 10^{-13} \mathrm{~cm}$ due to the fundamental nature of protons in the hadronic picture. We derive the quantity $\eta$ as the number of Planck areas $A_{\ell c}$ on the surface area $A_{p}$ of a proton

$$
\eta=\frac{A_{p}}{A_{\ell c}} .
$$

In this case, $\eta=4.716551 \times 10^{40}$. Multiplying by the Planck mass, we obtain 


$$
\eta_{\rho}=\eta m_{\ell}=1.026562 \times 10^{36} \mathrm{gm}
$$

or the holographic mass of the surface horizon of the proton. We then calculate $R$ or the number of PSUs within the proton volume $V_{p}$ utilizing equation (8), yielding $R=1.280404 \times 10^{60}$.

We can now examine the relationship between $\eta_{\rho}$ and $R$ and find

$$
m_{p^{\prime}}=2 \frac{\eta_{\rho}}{R}=1.603498 \times 10^{-24} \mathrm{gm}
$$

where $m_{p^{\prime}}$ is the holographic derivation of the mass of the proton. The result is a close approximation to the measured CODATA value for the proton mass $m_{p}=1.672622 \times 10^{-24} \mathrm{gm}$ with a $0.069 \times 10^{-24} \mathrm{gm}$ or $\sim 4 \%$ deviation from the CODATA value.

Therefore a simple reversal of the holographic "pixelation" relationship in equation (11) produces a close approximation to the rest mass of the proton; whereas the above geometric holographic gravitational mass (which is equivalent to the Schwarzschild solution) is generated by dividing the mass of PSUs in the interior by the number of PSUs on the surface, conversely the proton rest mass is extrapolated from the mass of PSUs on the surface divided by the number of PSUs in the interior. Clearly both equation (11) and it's inverse in equation (24) can be utilized to describe a relationship between the interior information to the screening on the surface horizon and is consistent with the dimensional reduction associated with the holographic approach. In the following sections we will clarify the nature of this relationship, which has significant implications to the gravitational coupling constant and confinement.

The usual method of determining the charge radius of the proton is to measure the Lamb shift of a bound proton-lepton system via spectroscopy. A prior method was to measure the Sachs electric form factor with a scattering experiment, such as electron-proton scattering. The Sachs form factors are the spatial Fourier transforms of the proton's charge distribution in the Breit frame [12]. Recently, in 2010, an international research team from the Paul Scherrer Institute (PSI) in Villigen (Switzerland) and scientists from the Max Planck Institute of Quantum Optics (MPQ) in Garching, the Ludwig-Maximilians-Universität (LMU) Munich and the Institut für Strahlwerkzeuge (IFWS) of the Universität Stuttgart (both from Germany), and the University of Coimbra, Portugal obtained measurements recently published in Nature of the spectrum of muonic hydrogen that found a significantly lower value of $r_{p}=0.84184 \times 10^{-13} \mathrm{~cm}$ [10] compared to the CODATA value of the proton charge radius. In the case of measuring the Lamb shift of a bound proton-muon system it was anticipated to reduce the error by an order of magnitude compared to measurements from proton-electron scattering and typical proton-electron spectroscopy [13]. While it did indeed reduce the error by an order of magnitude, the fact that the new measurement is five standard deviations from the CODATA value has raised significant questions about the implications of this new 
result on Quantum Electrodynamics, and so far no experimental errors have been found despite thorough scrutiny by the physics community [14-20].

We now proceed to calculate the rest mass of the proton as above, utilizing the new muonic hydrogen measured proton charge radius $r_{p}=0.84184 \times 10^{-13} \mathrm{~cm}$ and find $\eta=4.340996 \times 10^{40}, \quad \eta_{\rho}=9.448222 \times 10^{35} \mathrm{gm}$, and $R=1.130561 \times 10^{60}$. Again utilizing equation (24) we obtain

$$
m_{p^{\prime}}=2 \frac{\eta_{\rho}}{R}=1.6714213 \times 10^{-24} \mathrm{gm}
$$

This result is now a much closer approximation to the measured CODATA value for the proton mass $m_{p}=1.672622 \times 10^{-24} \mathrm{gm}$ with a $0.0012 \times 10^{-24} \mathrm{gm}$ or $\sim 0.07 \%$ deviation from the CODATA value. This extremely close result is supportive of the new muonic hydrogen measurement of the proton charge radius, and of our generalized holographic approach applied to the quantum scale. Considering that this method yields an exact solution to the gravitational mass of an object, we can now make a prediction of the precise radius of the proton from theoretical tenets. Assuming that the current CODATA mass measurement of the proton (which has been measured to a high level of precision empirically) is accurate, we can solve equation (25) for the radius of an object of mass $m_{p}=1.672622 \times 10^{-24} \mathrm{gm}$ by utilizing algebraic computations from the geometric consideration. Consequently

$$
m_{p^{\prime}}=2 \frac{\eta_{\rho}}{R}=2 \frac{\left(A / A_{\ell c}\right) m_{\ell}}{V / V_{\ell s}}
$$

Substituting equations (5) and (6) on the right side and canceling common terms we have

$$
=2 \frac{\left(4 \pi r^{2} / \pi \ell_{r}^{2}\right) m_{\ell}}{4 / 3 \pi r_{p}^{3} /\left(4 / 3 \pi \ell_{r}^{3}\right)}=2 \frac{\left(4 r^{2} / \ell_{r}^{2}\right) m_{\ell}}{r_{p}^{3} / \ell_{r}^{3}}
$$

and reducing to

$$
=\frac{8 m_{\ell}}{r_{p} / \ell_{r}}=\frac{8 \ell_{r} m_{\ell}}{r_{p}}
$$

Since $\ell_{r}=\ell / 2$, we can reduce this to

$$
m_{p^{\prime}}=4 \ell \frac{m_{\ell}}{r_{p}}
$$


Therefore the mass of the proton can be simply extrapolated from the relationship of the Planck length times the Planck mass divided by the proton charge radius. Again, as in section 2 we find a simple and elegant quantized solution to a fundamental physical quantity utilizing an intrinsic generalized holographic relationship.

We now can predict a precise radius for the proton, which we term $r_{p^{\prime}}$, from the CODATA value for the proton mass by inverting equation (29)

$$
r_{p^{\prime}}=4 \ell \frac{m_{\ell}}{m_{p}}=0.841236 \times 10^{-13} \mathrm{~cm}
$$

a difference of $0.000604 \times 10^{-13} \mathrm{~cm}$ from the muonic measurement of the proton charge radius of $r=0.84184(67) \times 10^{-13} \mathrm{~cm}$ and therefore falls within less than one standard deviation $0.00067 \times 10^{-13} \mathrm{~cm}$, or within their reported standard experimental error value [10]. More precise measurement may confirm this theoretical result.

\section{DETERMINING A FUNDAMENTAL GEOMETRIC MASS RATIO AND THE GRAVITATIONAL COUPLING CONSTANT}

As in section 2, we now replace $\ell$ and $m_{\ell}$ in equation (29) by their respective fundamental constant Planck unit definitions, to derive deeper meaning. Therefore, canceling terms and simplifying

$$
m_{p^{\prime}}=4 \ell \frac{m_{\ell}}{r_{p}}=\frac{4 \sqrt{\frac{\hbar G}{c^{3}} \frac{\hbar c}{G}}}{r_{p}}=\frac{4 \sqrt{\frac{\hbar^{2}}{c^{2}}}}{r_{p}}=\frac{4 \frac{\hbar}{c}}{r_{p}}=\frac{4 \hbar}{r_{p} c} .
$$

We rewrite the last term and multiply the numerator and denominator by $c / G$,

$$
=2 \frac{\hbar}{r_{p} c / 2}=2 \frac{\hbar c / G}{r_{p} c^{2} / 2 G}
$$

and since $m_{\ell}=\sqrt{\frac{\hbar c}{G}}$, we substitute

$$
=2 \frac{m_{\ell}^{2}}{r_{p} c^{2} / 2 G} .
$$

Here the Schwarzschild condition $m_{s}=r c^{2} / 2 G$ appears in the denominator which is equivalent to our holographic solution $m_{h}=r m_{\ell} / 2 \ell$. We can now write equation (33) as 


$$
m_{p^{\prime}}=2 \frac{m_{\ell}^{2}}{m_{h^{\prime}}} .
$$

This is a significant result as we now observe a direct relationship between the rest mass of the proton $m_{p}$, the Planck mass $m_{\ell}$, and the Schwarzschild mass or holographic gravitational mass $m_{h}$, which we denote as $m_{h^{\prime}}$ to indicate the holographic gravitational mass specific to the proton. Thus, the presence of a strong gravitational potential equivalent to the Schwarzschild mass in equation (34) relates the rest mass of the proton to our cosmological generalized holographic mass solution, confirming that the holographic principle, typically consistent with strong gravitational objects, is potentially involved in the strong field confinement environment on the femtometer scale due to Planck fluctuations. Here our generalized holographic approach has led us to a direct relationship between a cosmological gravitational solution and the Planck scale to produce the mass of a quantum object. From equation (11)

$$
m_{h}=\frac{R m_{\ell}}{\eta}
$$

where $R$ is the number of PSUs within the interior and $\eta$ is the number of PSUs on the surface horizon, we now clearly discern that both the holographic gravitational mass (equivalent to the Schwarzschild mass) and the rest mass of the proton are a consequence of the Planck mass $m_{\ell}$, and the geometrical considerations of Planck vacuum oscillators alone.

Although equation (35) has a simple and elegant form, we now explore a little further the algebra to better understand the geometric relationship between $m_{p^{\prime}}, m_{\ell}$ and $m_{h^{\prime}}$.

Starting from equation (34) and multiplying by $m_{h^{\prime}} / m_{h^{\prime}}$ we have

$$
m_{p^{\prime}}=2 \frac{m_{\ell}^{2}}{m_{h^{\prime}}}=2 \frac{m_{\ell}^{2}}{m_{h^{\prime}}^{2}} m_{h^{\prime}}
$$

Expanding $m_{h^{\prime}}$ in the denominator with equation (35) and rearranging terms we have

$$
=2 \frac{m_{\ell}^{2}}{\left(\frac{R m_{\ell}}{\eta}\right)^{2}} m_{h^{\prime}}=2\left(\frac{\eta m_{\ell}}{R m_{\ell}}\right)^{2} m_{h^{\prime}} .
$$

We now express this in terms of $\eta_{\rho}$ and $R_{\rho}$ 


$$
m_{p^{\prime}}=2\left(\frac{\eta_{\rho}}{R_{\rho}}\right)^{2} m_{h^{\prime}}
$$

where $\eta_{\rho}$ is the mass of PSUs on the surface horizon and $R_{\rho}$ is the mass of PSUs in the interior volume as in equation (10). Here the geometric mass relationship clearly emerges. Significantly, the rest mass of the proton is generated by the square of the simple mass relationship of the surface mass of PSUs to the interior mass of PSUs multiplied by the holographic gravitational mass of the proton. Of course we can also express this relationship in terms of dimensionless quantities. We divide by $m_{\ell}$ in the numerator and denominator

$$
m_{p^{\prime}}=2\left(\frac{\eta_{\rho} / m_{\ell}}{R_{\rho} / m_{\ell}}\right)^{2} m_{h^{\prime}}
$$

yielding

$$
=2\left(\frac{\eta}{R}\right)^{2} m_{h^{\prime}} .
$$

Yet, another step can be taken to further elucidate the nature of the relationship by expanding $m_{h^{\prime}}$ utilizing equation (9)

$$
=2\left(\frac{\eta}{R}\right)^{2} \frac{R}{\eta} m_{\ell}
$$

which reduces to

$$
m_{p^{\prime}}=2 \frac{\eta}{R} m_{\ell}
$$

which can be converted back to a mass only expression by multiplying the dimensionless quantities by $m_{\ell}$, yielding

$$
m_{p^{\prime}}=2 \frac{\eta_{\rho}}{R_{\rho}} m_{\ell}
$$

The relationships between the proton mass, the Planck mass and the holographic gravitational mass clearly emerge from this algebraic sequence of equations. One of the most significant challenges of modern physics has been to find a comprehensive framework to explain the significant discrepancy between the relatively large Planck mass, the mass of the proton, and the gravitational force or what is known as the hierarchy problem. Frank 
Wilczek, whose fundamental contribution of asymptotic freedom to the strong interaction theory, states 'We see that the question it poses is not, 'Why is gravity so feeble?' but rather, 'Why is the proton's mass so small?' For in natural (Planck) units, the strength of gravity simply is what it is, a primary quantity, while the proton's mass is the tiny number..." [21]

Here the hierarchy problem between the Planck mass and the proton rest mass is resolved as we clearly demonstrate that the rest mass of the proton is a function of the Planck vacuum oscillators holographic surface to volume geometric relationship of spacetime, the energy levels of which include the gravitational mass-energy $m_{h^{\prime}}$ derived from the same primary quantity of Planck entities. We express the relationship of the proton surface horizon to its volume Planck oscillators as a fundamental constant we term $\phi$

$$
\phi=\frac{\eta}{R}=\frac{\eta_{\rho}}{R_{\rho}}=3.839682 \times 10^{-20}
$$

which appears as a fundamental geometric ratio from equations (38) to (43), whether in dimensionless quantities or in mass ratios. The inverse relationship

$$
\frac{1}{\phi}=\frac{R}{\eta}=\frac{R_{\rho}}{\eta_{\rho}}=2.604382 \times 10^{19}
$$

is clearly seen in equation (41) where $m_{h^{\prime}}$ is fully expanded in its holographic expression from equation (9) of section 2. Therefore, $\phi$ and its inverse relate the gravitational curvature of a Schwarzschild metric to the quantum scale so that

$$
m_{p^{\prime}}=2 \phi^{2} \frac{1}{\phi} m_{\ell}=2 \phi^{2} m_{h^{\prime}}
$$

and relates the proton rest mass to the Planck mass

$$
m_{\ell}=\frac{m_{p^{\prime}}}{2 \phi}
$$

and of course the Planck mass to the holographic gravitational mass is $\phi$

$$
m_{\ell}=\phi m_{h^{\prime}}
$$

Consequently $\phi$ acts as a fundamental constant relating the background Planck vacuum fluctuation field to the cosmological and quantum scale where it may be the source of confinement so that scaling from the proton rest mass to the Planck mass requires a proportional mass-energy conversion of $2 \phi$ while from the Planck mass to the holographic 
gravitational mass requires a factor of $\phi$, which yields a total scaling from the proton rest mass to the holographic gravitational mass of

$$
2 \phi^{2}=2.948632 \times 10^{-39} \text {. }
$$

Exploring the $\phi$ relationships relative to quantum gravity confinement, we utilize equation (47), and we determine

$$
m_{p^{\prime}}=2 \phi m_{\ell}=2 \phi \sqrt{\frac{\hbar c}{G}}
$$

Squaring both sides

$$
m_{p^{\prime}}^{2}=4 \phi^{2} \frac{\hbar c}{G}
$$

Multiplying both sides by $\frac{G}{\hbar c}$ we have

$$
4 \phi^{2}=\frac{G m_{p^{\prime}}^{2}}{\hbar c}=\frac{G m_{p^{\prime}} m_{p^{\prime}}}{\hbar c} .
$$

Where $4 \phi^{2}=5.897264 \times 10^{-39}$ is the exact value for the coupling constant between gravitation and confinement at the proton scale or the strong interaction. The typical computation given for the gravitational coupling constant is

$$
\frac{F_{g}}{F_{s}}=\frac{F_{g}}{F_{e}} \frac{F_{e}}{F_{s}}=\frac{G m_{p} m_{p} / r^{2}}{e^{2} / r^{2}} \alpha=\frac{G m_{p}^{2}}{e^{2}} \alpha=5.905742 \times 10^{-39}
$$

where $e$ is the elementary charge and $\alpha$ is the fine structure constant. Note that the slightly different value of equation (53) from $4 \phi^{2}$ of equation (52) is due to our utilization of the 2010 muonic measurement of the radius of the proton, and that utilizing our predicted radius $r_{p^{\prime}}$ from equation (30) yields the exact value.

Hence the gravitational force coupling constant is computed directly from the geometric relationship of the Planck oscillator surface tiling to the interior volume oscillations of the proton which as well clearly relates the Planck mass to the proton rest mass, and the $2 \phi^{2}$ ratio of the proton mass to the holographic gravitational mass or the Schwarzschild mass. Consequently, the unifying energy required for confinement is generated by holographic derivations directly from first principles of simple geometric Planck vacuum fluctuation relationships. Furthermore, the rest mass of the proton is computed without requiring the complexities introduced by a Higgs mechanism, which also utilizes a non-zero vacuum expectation value, but which only predicts 1 to 5 percent of the mass of baryons, and in 
which the Higgs particle mass itself is a free parameter [22]. The current QCD approach accounts for the remaining mass of the proton by the kinetic back reaction of massless gluons interacting with the confining color field utilizing special relativity to determine masses. Yet it is critical to note that after almost a century of computation, there is still no analytical solution to the Lattice QCD model for confinement. This problem is thought to be one of the most obscure processes in particle physics and a Millennium Prize Problem from the Clay Mathematics Institute has been issued to find a resolution [23,24]. Since there is no analytical solution to LQCD and no framework for the energy source necessary for confinement, associating the remaining mass of the proton to the kinetic energy of massless gluons is based on tenuous tenets.

Our results demonstrate that the holographic gravitational mass-energy of the proton $m_{h^{\prime}}$ is the unification energy scale for hadronic confinement and that the mass of nucleons is a direct consequence of vacuum fluctuations. Keeping in mind that a neutron quickly decays into a proton when free of the nucleus, we have therefore addressed the fundamental nature of the nucleon by deriving the proton rest mass and the confining force from holographic considerations. In future publications we will address the confinement string-like gluon jet flux tube structures of the QCD vacuum model as potentially arising from high curvature within the spacetime Planck vacuum collective behavior background, acting as vortices near the holographic screen topological horizon. This will be addressed utilizing an extended center vortex picture which has been significantly developed by 't Hooft [25] and in which the surface area of a Wilson loop is related to a confining force. In the next section, we explore the energy and angular frequency associated with our model and we compute the gravitational potential range of our confining force utilizing special relativity.

\section{FREQUENCY, ENERGY AND THE YUKAWA POTENTIAL}

From equations (29) and (47) we have

$$
m_{p^{\prime}}=2 \phi m_{\ell}=4 \ell \frac{m_{\ell}}{r_{p}}
$$

Dividing by $2 m_{\ell}$ on both sides we find

$$
\phi=\frac{2 \ell}{r_{p}}
$$

or

$$
r_{p}=\frac{2 \ell}{\phi}
$$

Calculating Einstein's mass-energy equivalence for the proton we have

$$
E_{p}=m_{p^{\prime}} c^{2}
$$

From equation (47) we can then write 


$$
=2 \phi m_{\ell} c^{2}
$$

where $m_{\ell} c^{2}$ is the Planck energy. Now we expand the terms

$$
=2 \phi \sqrt{\frac{\hbar c^{5}}{G}}=2 \phi \sqrt{\frac{\hbar}{\hbar} \frac{\hbar c^{2} c^{3}}{G}}=2 \phi \sqrt{\frac{\hbar^{2} c^{2} c^{3}}{\hbar G}}=2 \phi \hbar c \sqrt{\frac{c^{3}}{\hbar G}}=\frac{2 \phi \hbar c}{\sqrt{\frac{\hbar G}{c^{3}}}}=\frac{2 \phi \hbar c}{\ell} .
$$

From equation (56) it follows that

$$
=\frac{4 \phi \hbar c}{2 \ell}=\frac{4 \hbar c}{\frac{2 \ell}{\phi}}=\frac{4 \hbar c}{r_{p}} .
$$

Given that $\hbar=\frac{h}{2 \pi}$, then

$$
E_{p}=\frac{4 h c}{2 \pi r_{p}}=\frac{4 h c}{C_{p}}=4 h f_{p}
$$

Thus we have obtained an expression for the energy where $C_{p}=2 \pi r_{p}$ is the circumference of the proton and the angular frequency $f_{p}=\frac{c}{C_{p}}$. Therefore the energy of such a system can be written in terms of $\hbar$ as $E_{p}=8 \pi \hbar f_{p}$ which yields a frequency

$$
f_{p}=\frac{E_{p}}{8 \pi \hbar}=\frac{E_{p}}{4 h}=5.667758 \times 10^{22} h z
$$

characteristic to high-energy nuclear gamma emission, and a period of

$$
t_{p}=\frac{1}{f_{p}}=1.764366 \times 10^{-23} \mathrm{sec}
$$

where $10^{-23} \mathrm{sec}$ is typically given as the interaction time of the strong force [26]. From equation (58) we find that $2 \phi$ multiplied by the Planck energy yields an angular frequency with a period of $t_{p}$, which is the time it takes for a particle to decay via the strong interaction. Hence from the generalized holographic geometric relations of Planck entities, we have derived clear quantum gravitational mass-energy formulations that define the characteristics 
of the strong nuclear force, such as the energies to produce it from gravitational coupling and its interaction time.

Yet, the short range of the nuclear force as defined by the Yukawa potential demands that the force strength drops off at an exponential rate close to the horizon where $r=r_{p}$. To explore this force strength to radius relation in our approach, we begin by refining our derivation from reference [27] where we theorize that the difference between the Schwarzschild energy potential and the rest mass of the proton may be the result of mass dilation near the horizon where velocity is relativistic. Therefore, we begin with the known relativistic mass dilation expression

$$
M=\frac{m_{0}}{\sqrt{1-\frac{v^{2}}{c^{2}}}}
$$

where $m_{0}$ is a rest mass and $M$ is the dilated mass and $v$ is the velocity. Solving for $\frac{v}{c}$, we find

$$
\frac{v}{c}=\sqrt{1-\left(\frac{m}{M}\right)^{2}}
$$

Substituting $m_{0}=m_{p}$ and $M=m_{h}$

$$
\frac{v}{c}=\sqrt{1-\left(\frac{m_{p^{\prime}}}{m_{h^{\prime}}}\right)^{2}}=\sqrt{1-4 \phi^{4}} .
$$

Therefore the dilated mass-energy yielding the Schwarzschild unifying energy potential occurs at $\frac{v}{c}$ extremely close to 1 . We compute the result and examine how close $v$ is to $c$ and find

$$
1-\frac{v}{c}=4.347214 \times 10^{-78}
$$

That is, the Schwarzschild energy potential is reached when $v$ is $4.34 \times 10^{-78}$ less than $c$, which can be computed as well, to a high degree of accuracy, to be $2 \phi^{4}$. We now seek an expression for $v$ as a function of $r$ utilizing an orbital velocity formula. Our purpose is to identify velocities at the Schwarzschild horizon or the holographic horizon described in earlier sections. The use of relativistic velocity equations produces results describing velocities at the photon sphere or the ergosphere in the case of the Kerr Metric where the ergosurface is situated at 1.5 times the Schwarzschild radius at the equator (the photon 
sphere) and is oblate so that the poles are coincident with the Schwarzschild surface. We note that the relativistic photon sphere solution corresponds closely with the Compton wavelength of the proton. However, for our purpose in this work our intent is to compute the velocity at the Schwarzschild surface or holographic surface rather than the ergosphere. For that purpose a simple semi-classical form can be utilized. Therefore

$$
v(r)=\sqrt{2 a r}=\sqrt{2 \frac{G m}{r^{2}} r}=\sqrt{\frac{2 G m}{r}}
$$

and multiplying by $c^{2}$ in the numerator and denominator and utilizing the Schwarzschild radius equation

$$
=c \sqrt{\frac{2 G m}{r c^{2}}}=c \sqrt{\frac{r_{s}}{r}} .
$$

Substituting $v(r)$ into the mass dilation equation (64) we have

$$
M=\frac{m}{\sqrt{1-\frac{[v(r)]^{2}}{c^{2}}}}=\frac{m}{\sqrt{1-\frac{c^{2} r_{s}}{r c^{2}}}}=\frac{m}{\sqrt{1-\frac{r_{s}}{r}}}
$$

Substituting $m_{h^{\prime}}$ for $m$ and $r_{p}$ for $r_{s}$, we can derive that the radius at which the unification energy $m_{h^{\prime}}=5.668464 \times 10^{14} \mathrm{gm}$ is achieved due to mass dilation can be computed as

$$
r=r_{p} \frac{m_{h^{\prime}}{ }^{2}}{m_{h^{\prime}}{ }^{2}-m_{p^{\prime}}{ }^{2}}=r_{p} \frac{m_{h^{\prime}}{ }^{2}}{\left(m_{h^{\prime}}{ }^{2}-\left(2 \phi^{2} m_{h^{\prime}}\right)^{2}\right)}=r_{p} \frac{m_{h^{\prime}}{ }^{2}}{m_{h^{\prime}}{ }^{2}\left(1-4 \phi^{4}\right)}=\frac{r_{p}}{\left(1-4 \phi^{4}\right)}
$$

or the dimensionless quantity $\left(r-r_{p}\right) / r_{p}=8.694428 \times 10^{-78}$. Consequently we can assert for all intent and purposes, that the Schwarzschild mass occurs at or extremely close to the horizon. We now compute the mass dilation from the velocity found at $\ell$ from $r_{p}$ utilizing equation (70) and find

$$
m_{p d}^{\ell}=\frac{m_{p^{\prime}}}{\sqrt{1-\frac{r_{p}}{r_{p}+\ell}}}=\sqrt{\frac{2}{\phi} \frac{\left(r_{p}+\ell\right)}{r_{p}}} m_{p^{\prime}}=1.206294 \times 10^{-14} \mathrm{gm}
$$

where $m_{p d}^{\ell}$ is the dilated mass at one Planck length from $r_{p}$. Evidently an asymptotic drop of the dilated mass-energy $m_{h^{\prime}}$ occurs, reducing by some 28 orders of magnitude within one 
Planck length from the horizon. We note that $\frac{m_{p d}^{\ell}}{2}$ is equivalent to the geometric mean $\sqrt{m_{p^{\prime}} m_{h^{\prime}}}$ between the Planck mass and the rest mass of the proton, which may represent a harmonic relationship between $m_{p d}^{\ell}$ and $m_{p^{\prime}}$.

We now utilize equation (70) to compute mass dilation as a function of radius, which we convert to a gravitational energy potential $\mathrm{Gm} / r$. We graph our results and compare them with the Yukawa potential, see Fig. 1a.

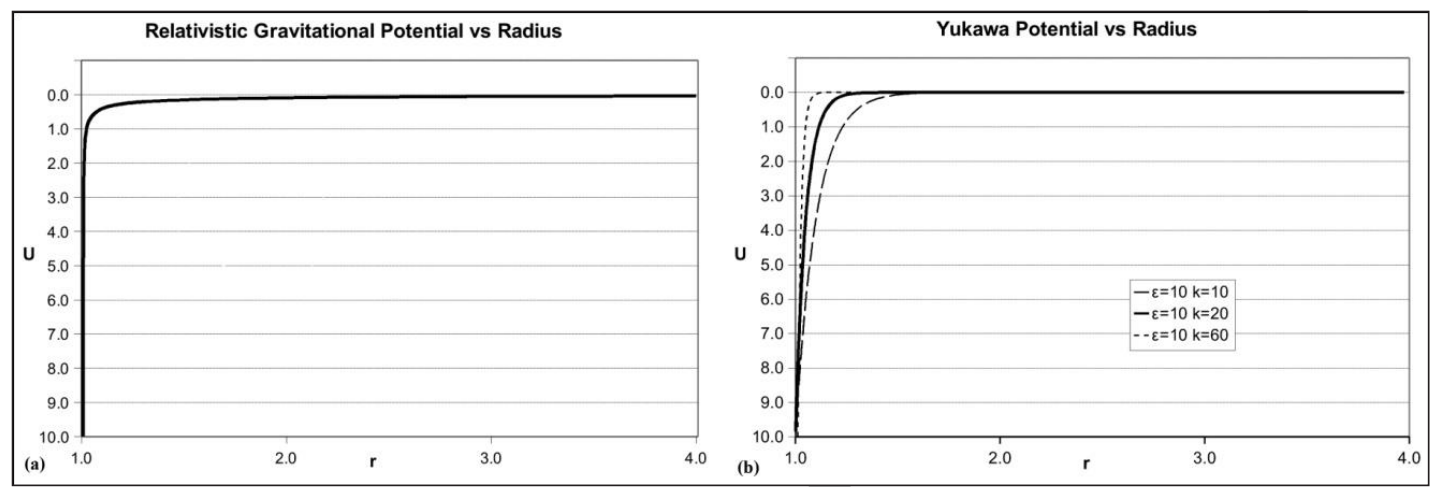

Fig. 1. (a) The relativistic gravitational potential $U$ resulting from mass dilation near the horizon $r_{p}$. (b) The Yukawa potential $\cup$ typically given as the short range energy potential of the strong force where $\mathcal{E}$ is the hard-core surface potential and $k$ is the
inverse screening length (inverse Debye length)

From Fig. 1(a) we find that the gravitational potential from the mass dilation of a proton due to the angular velocity of an accelerated frame generates an asymptotic curve with a force potential drop-off as a function of $r$ characteristic of the short range force of nuclear confinement equivalent to the Yukawa potential in Fig. 1(b). Therefore, we have derived a relativistic source for the confining energy with a quantum gravitational potential equivalent to the unification energy of a Schwarzschild mass or the holographic gravitational mass of the proton $m_{h^{\prime}}$, yielding a gravitational coupling with a Yukawa-like short range, and the appropriate interaction time of the strong force $t_{p}$, resulting in an analytical solution to confinement. These results are derived from first principles and classical considerations alone, with zero free parameters or hidden variables, and extend our generalized holographic solution to generate a complete picture of confinement whether at the quantum scale or the cosmological scale of black holes. Furthermore, considerations of equations (38) and (43), where the rest mass of the proton is derived from relationships of Planck oscillators PSUs of an energetic structured vacuum at the holographic horizon, may provide us with a source for mass. This is analogous to the non-zero vacuum expectation value of the Higgs field where the Yukawa interaction describes the coupling between the Higgs mechanism and massless quark and lepton fields or fermions. However, this Higgs mechanism only accounts for a small percentage of the mass of baryons where the rest is 
thought to be due to the mass added by the kinetic energies of massless gluons inside the baryons. Our generalized holographic model accounts for all of the rest mass of protons and the energy of confinement in addition to predicting the mass of cosmological objects directly out of geometric considerations of the energetic vacuum.

\section{CONCLUSION}

We have generalized the holographic principle to considerations of spherical tiling of Planck vacuum fluctuations within volumes as well as on horizon surfaces. From these discrete spacetime quantization relationships we extract the Schwarzschild solution to Einstein's field equations, generating a novel quantized approach to gravitation. We apply this resulting quantum gravitational method to the nucleon to confirm its relevance at the quantum scale and we find values for the rest mass of the proton within $0.069 \times 10^{-24} \mathrm{gm}$ or $\sim 4 \%$ deviation from the CODATA value and $0.0012 \times 10^{-24} \mathrm{gm}$ or $\sim 0.07 \%$ deviation when the 2010 muonic radius measurement is utilized. As a result, we predict a precise proton charge radius utilizing our holographic method which falls within the reported experimental uncertainty for the muonic measurement of the proton charge radius. More precise experiments in the future may confirm our predicted theoretical proton charge radius.

We determine a fundamental constant $\phi$ defined by the mass ratio of vacuum oscillations on the surface horizon to the ones within the volume of the proton. As a result, clear relationships emerge between the Planck mass, the rest mass of the proton, and the Schwarzschild mass of the proton or what we term the holographic gravitational mass. Furthermore, we find that $4 \phi^{2}$ generates the coupling constant between gravitation and the strong interaction, thus defining the unification energy for confinement. We also derive the energy, angular frequency, and period for such a system utilizing our holographic approach and find that the frequency is the characteristic gamma frequency of the nucleon and the period is on the order of the interaction time of particle decay via the strong force. Finally, we calculate the mass dilation due to velocity as a function of radius and plot the resulting gravitational potential range. We find the range to be a close correlation to the Yukawa potential typically utilized to illustrate the sharp drop-off of the confining force. In future work we will examine the application of this approach to more complex systems. We will consider as well some of the seminal work done in defining maximal particle momentum and it's applicability to our approach [28].

In this paper, we demonstrate that a quantum gravitational framework of a discrete spacetime defined by spherical Planck vacuum oscillators can be constructed which applies to cosmology and quantum scale. Our generalized holographic method utilizes zero free parameters and is generated from simple geometric relationships and algebra, yielding precise results for significant physical properties. In the words of Einstein, "One can give good reasons why reality cannot at all be represented by a continuous field. From the quantum phenomena it appears to follow with certainty that a finite system of finite energy can be completely described by a finite set of numbers (quantum numbers). This does not seem to be in accordance with a continuum theory and must lead to an attempt to find a purely algebraic theory for the representation of reality." [29]. 


\section{ACKNOWLEDGEMENTS}

The author would like to express sincere gratitude and great appreciation to Stephen Bard, David Gerow, Dr. Michael Hyson and Dr. Elizabeth Rauscher for their support and fellowship which provided substantial assistance for the redaction and completion of this manuscript.

\section{COMPETING INTERESTS}

Author has declared that no competing interests exist.

\section{REFERENCES}

1. Schwarzschild K. Uber das Gravitationsfeld eines Massenpunktes nach der Einsteinschen Theorie. Sitzungsberichte der Deutschen Akademie der Wissenschaften zu Berlin, Klasse fur Mathematik, Physik, und Technik; 1916.

2. Schwarzschild K. Uber das Gravitationsfeld einer Kugel aus inkompressibler Flussigkeit nach der Einsteinschen Theorie. Sitzungsberichte der Deutschen Akademie der Wissenschaften zu Berlin, Klasse fur Mathematik, Physik, und Technik; 1916.

3. Wheeler JA. Geons. Phys. Rev. 1955;97:511-536.

4. Ford KW, Wheeler JA. Geons, Black Holes and Quantum Foam - a life in Physics. New York. W. W. Norton and Co; 1998.

5. Genet C, Lambrecht A, Reynaud S. Casimir Effect and Vacuum energy. Laboratoire Kastler Brossel UPMC/ENS/CNRS case 74, Campus Jussieu, F75252, Paris Cedex 05. arXiv:quant-ph/0210173v1. 2002;1-10.

6. Bekenstein JD. Black holes and entropy. Physical Review D7. 1973;8:2333-2346.

7. Hawking S. Particle Creation by Black Holes. Comm. Math. Phys. Springer-Verlag. 1975;(43):199-220.

8. 't Hooft G. Dimensional reduction in quantum gravity. arXiv:gr-qc/9310026v2. 2009;20:1-13.

9. Susskind L. The world as a hologram. J. Math. Phys. arXiv:hep-th/9409089, 10.1063/1.531249. 1994;1-34.

10. Pohl R, Antognini A, Nez F, Amaro FD, Biraben F, Cardoso JMR, et. al. The size of the proton. Nature. 2010;466:213-216.

11. 't Hooft G. The Holographic Principle. arXiv:hep-th/0003004v2. 2000;1-15.

12. Sachs RG. High-energy behavior of nucleon electromagnetic form factors. Phys. Rev. 1962;126:2256-2260.

13. Pachucki K. Theory of the Lamb shift in muonic hydrogen. Phys. Rev. 1996;A 53(4):2092-2100.

14. Barger V, Chiang CW, Keung WY, Marfatia D. Proton size anomaly. Phys. Rev. Lett. 10.1103/PhysRevLett.106.153001. 2011;106(15):4.

15. Tucker-Smith D, Yavin I. Muonic hydrogen and MeV forces. Physical Review D. 10.1103/PhysRevD.83.101702. 2011;10(83):5.

16. Batell B, McKeen D, Pospelov M. New Parity-Violating Muonic Forces. Phys. Rev. Lett. 03/2011; DOI:10.1103/PhysRevLett.107.011803. 2011;107(1):011803.

17. Arrington J. New measurements of the proton's size and structure using polarized photons. Proceedings of plenary talk at CIPANP 2012, St Petersburg, FL. arXiv:1208.4047. 2012;8.

18. Walcher T. Some issues concerning the proton charge radius puzzle. arXiv:1207.4901v2. 2012;8. 
19. Carlson C, Rislow B. New Physics and the Proton Radius Problem. arXiv: 1206.3587v2. 2012;6.

20. Kelkar N, Daza F, Nowakowski M, Determining the size of the proton, Nuclear Physics. 2012;B(864):382-398.

21. Wilczek F. Scaling Mount Planck I: A View from the Bottom. Physics Today. 2001:1213.

22. Wilczek F. Origins of Mass. Invited review for the Central European Journal of Physics. arXiv: hep-ph/1206.7114. 2012;1-35.

23. Carlson J, Jaffe A, Wiles A. The Millenium Prize Problems. American Mathematical Society. Cambridge, MA; 2006.

24. Jaffe A, Witten E. Quantum Yang-Mills Theory from the Millenium Prize Problems. American Mathematical Society. 2006;129-152.

25. t'Hooft G. On the Phase Transition Towards Permanent Quark Confinement. Nuclear Physics. 1978;B138(1):42.

26. Choppin GR, Liljenzin J-O, Rydberg J. Radiochemistry and Nuclear Chemistry. Butterworth-Heinemann. 2001;1:323-288.

27. Haramein N. The Schwarzschild Proton. International Journal of Computing Anticipatory Systems. American Institute of Physics Conference Proceedings. 2009;1303:95-100.

28. Nozari K, Etemadi A. Minimal length, maximal momentum and Hilbert space representation of quantum mechanics. Phys. Rev. D. 10.1103/PhysRevD.85.104029. 2012;85(10):12.

29. Einstein A. The Meaning of Relativity. Methuen. 1956;(6):169-170.

(C) 2013 Haramein; This is an Open Access article distributed under the terms of the Creative Commons Attribution License (http://creativecommons.org/licenses/by/3.0), which permits unrestricted use, distribution, and reproduction in any medium, provided the original work is properly cited.

Peer-review history:

The peer review history for this paper can be accessed here: http://www.sciencedomain.org/review-history.php?iid=224\&id=4\&aid=1298 